\title{
The effect of a modulated flux on the growth of thin films
}

\author{
Pablo Jensen* and Bernd Niemeyer \\ Département de Physique des Matériaux, Université Claude Bernard Lyon-1, 69622 Villeurbanne \\ Cédex, France
}

\begin{abstract}
Thin films are usually obtained by depositing atoms with a continuous flux. We show that using a chopped flux changes the growth and the morphology of the film. A simple scaling analysis predicts how the island densities change as a function of the frequency of the chopped flux in simple cases where aggregation is irreversible. These predictions are confirmed by computer simulations. We show that the model can be used to obtain information on the diffusion or the evaporation of the adatoms. The model is also useful to understand the growth of thin films prepared by pulsed sources.
\end{abstract}

Typeset using REVTEX 
One of the main interests of usual deposition techniques such as Molecular Beam Epitaxy [1] is that the structure of the deposited films is to a large extent determined by kinetic factors, as opposed to thermodynamic equilibrium. This allows to "play games" [2] with the different growth parameters (incident flux of particles, diffusion coefficient of an adatom ...) in order to obtain different film morphologies. A simple example is given by the quantity of islands grown on a substrate at low enough temperatures : it is known that the number of islands at saturation is given by $(F / D)^{1 / 3}$ [3 5 where $F$ is the incident flux and $D$ the diffusion coefficient. Then, by increasing the flux or decreasing the diffusion constant (by lowering the substrate temperature), one can adjust the saturation number of islands grown on the substrate. In this sense, each kinetic factor is a "handle" on the system, allowing to control the morphology of the films. We introduce in this Letter a new kinetic handle, which should enable a larger control over film growth : the chopping of the incident flux. We note that this flux modulation is intrinsic to other deposition techniques such as cluster laser vaporization (the laser is pulsed [6]). It is therefore important to understand how growth proceeds in the presence of a modulated flux if one is to be able to interpret experiments performed in these conditions. For example, one may wonder whether the usual growth theories [3 5] can be used by replacing the continuous flux by the average value of the chopped flux over a cycle. In the following, we will show that this is not the case, and that the growth of the film is profoundly changed by the modulation of the incident flux for the case of growth with irreversible aggregation (critical island size 1, see [3 5, 7, 9]. Conversely, we show what kind of information can be derived from experiments carried under these conditions.

The basic idea of our method is that if instead of using a continuous flux we use a chopped flux to grow a film, the number of islands formed on a substrate will depend on the chopping frequency $f$ and on $d$, the fraction of the period the flux is "on" (see Fig. [1). This dependence is due to the fact that the free particle concentration on the surface does not reach its steady state concentration instantaneously, but only after a time which we will call $\tau_{m}$. Then, if the timescale of the chopping $(1 / \mathrm{f})$ is much smaller than $\tau_{m}$, the system 
only sees the average flux. In the contrary case, everything happens as if the instantaneous flux was used instead. Then, there will be a transition from one behaviour to the other at a chopping frequency close to $1 / \tau_{m}$. We discuss this in detail in next section in the case of growth without desorption: the corresponding analysis in presence of evaporation is briefly presented afterwards.

Presentation of the method: Fig 1 shows schematically the time evolution of the free adatom (monomer) concentration in presence of a modulated flux. We assume here, for simplicity, that adatom-adatom aggregation is irreversible and that only monomers can move on the surface [3 5, 179]. Then, the important timescale for growth is given by the time needed for monomers to reach their steady state concentration or to disappear. Since, in the absence of evaporation, the monomers disappear mainly by diffusing randomly until they aggregate with an island [3], their mean lifetime on the surface is given by $\tau_{m} \sim l^{2} / D$ where $2 l$ is the mean distance between islands and $D$ the diffusion constant of the monomers. We obtain $\tau_{m} \sim 1 /(D N)$ where $N$ is the island concentration. We can predict three regimes of behaviour, depending on the relative magnitude of $\tau_{m}, \mathrm{~d} / \mathrm{f}$ and $1 / \mathrm{f}$.

For low chopping frequencies $\left(\tau_{m} \ll d / f\right)$, the monomer concentration reaches its steady state concentration $\rho_{s s}=F \tau_{m}$ almost instantaneously in the timescale of a period. After the flux is turned off, the monomer concentration goes back to 0 also almost instantaneously $\left(\tau_{m} \ll 1 / f\right)$. Then, between two successive "flux on" periods, nothing happens since only the monomers can move, and there is no monomer left. Therefore, growth proceeds as if we had a continuous flux $F_{i}$ and the island concentration at saturation for low frequencies $N_{l f}$ satisfies the well-known result $N_{l f} \sim\left(F_{i} / D\right)^{\chi}$, with $\chi \sim 0.36$ for fractal islands [3].

When the frequency increases $\left(d / f \ll \tau_{m}\right)$, the monomer concentration cannot attain its steady state concentration during the "flux on" part of the cycle : $\rho(t=d / f) \sim F_{i} d / f \ll \rho_{s s}$. Since we are interested in $d \ll 1, \rho$ vanishes before next cycle, and the average of the monomer concentration over a cycle is smaller than in the preceding regime, leading to a smaller island density. More precisely, the increase of island density during 
a cycle is given by : $\Delta N=\int_{\text {cycle }} D \rho^{2}(t) d t$. Using $\rho(t)=\left(F_{i} d / f\right) e^{-t / \tau_{m}}$, we obtain $\Delta N \sim D \int_{d / f}^{\infty}\left(F_{i} d / f\right) e^{-2 t / \tau_{m}} \sim 1 / 2\left(F_{i} d / f\right)^{2} *\left(D \tau_{m}\right)$. Since $\tau_{m} \sim 1 / D N$, we obtain $\Delta\left(N^{2}\right) \sim\left(F_{i} d / f\right)^{2}$. We have checked that, as in the case of a continuous flux [3, 10, the saturation island density is reached for a surface coverage of about $15 \%$. Then, the total number of cycles needed to reach saturation is $n_{c} \sim f /\left(6 F_{i} d\right)$. Since $\Delta\left(N^{2}\right)$ is a constant over the cycles, we simply multiply $\Delta\left(N^{2}\right)$ by the number of cycles to obtain $N_{i}$ the saturation island density in this intermediate regime : $N_{i} \sim\left(F_{i} d / f\right)^{1 / 2}$. We note that here the saturation island density does not depend on the diffusion constant. This regime is limited by the fact that if $\tau_{m} \sim 1 / f$, the monomer concentration no longer fully decays when the next "on" cycle begins. The monomer concentration slowly increases, reaching its steady state value after several cycles.

We then cross to the regime of high frequencies $\left(\tau_{m} \gg 1 / f\right)$, where many deposition cycles are carried out during the monomer equilibration, and the system only sees the average flux $F_{a v}$. Then the island concentration at saturation for high frequencies $N_{h f}$ satisfies $N_{h f} \sim\left(F_{a v} / D\right)^{\chi}=d^{\chi} N_{l f} \ll N_{l f}$.

Measuring the evaporation time: We briefly treat the case of film growth in presence of significant evaporation of monomers from the surface (for a more detailed treatment in the presence of a continuous flux, see [10]). In this case, the lifetime of the adatoms is no longer determined by the aggregation with islands but rather by random evaporation after a mean time $\tau_{e}$ [4.5.,10]. We study here the dependence of the island density $N^{e}$ at a fixed amount of deposited matter as a function of the modulation frequency. This quantity is not necessarily the saturation island density but it is easier to measure experimentally when evaporation is important. As in the non evaporation case, three different regimes will be scanned as the frequency increases.

For low chopping frequencies $\left(\tau_{e} \ll d / f\right)$, the monomer concentration reaches its steady state concentration $\rho_{s s}=F \tau_{e}$ and vanishes almost instantaneously in the timescale of a period. Therefore, during a cycle, the island density increases according to $d N^{e} / d t \sim D \rho^{2} \sim$ 
$D F_{i}^{2} \tau_{e}^{2}$, leading to $\Delta N^{e}($ per cycle $)=D F_{i}^{2} \tau_{e}^{2} d / f$. The island density for fixed $M=F_{a v} t$ in this regime is therefore $N_{l f}^{e} \sim D F_{i} \tau_{e} M$ since the number of cycles is $n_{c}=f M /\left(d F_{i}\right)$.

When the frequency increases $\left(d / f \ll \tau_{e}\right)$, the monomer concentration cannot attain its steady state concentration during the "flux on" part of the cycle : $\rho(t=d / f) \sim F_{i} d / f \ll \rho_{s s}$. Again, the increase of island density during a cycle is given by : $\Delta N^{e}=\int_{\text {cycle }} D \rho^{2}(t) d t \sim$ $1 / 2\left(F_{i} d / f\right)^{2} D \tau_{e}$. Counting the number of cycles as before leads to an island density in this intermediate regime $N_{i}^{e} \sim D F_{i} d \tau_{e} M f^{-1}$. As in the case of complete condensation, this regime is limited by the fact that if $\tau_{e} \sim 1 / f$, the monomer concentration no longer fully decays when the next "on" cycle begins. Then, the monomer concentration slowly increases, reaching its steady state value after several cycles.

In the regime of high frequencies $\left(\tau_{e} \gg 1 / f\right)$, many deposition cycles are carried out during the monomer equilibration, and the system only sees the average flux $F_{a v}$. Using the result obtained in the low frequency case (see also Ref. [10]), the island concentration at saturation for high frequencies can be written as $N_{h f}^{e} \sim D F_{a v} \tau_{e} M=d N_{l f}^{e} \ll N_{l f}^{e}$.

Computer simulations : Monte-Carlo simulations of the growth of thin films by a continuous flux have been independently implemented by several groups with small variations [11 13. To simulate the growth in the presence of a chopped flux, we introduce the four following physical processes :

(1) Deposition. Particles are deposited at randomly-chosen positions of the surface at a flux $F_{i}$ during the "on" fraction of a cycle $(d \ll 1)$. During the rest of the cycle, no particle reaches the surface (see Fig 1). The average flux reaching the surface is thus $F_{a v}=d F_{i}$.

(2) Diffusion. Each diffusion time $\tau$, all monomers are chosen at random and moved in a random direction by one lattice site.

(3) Evaporation. Isolated adatoms can evaporate off the surface at a constant rate. We denote by $\tau_{e}$ the mean lifetime of a free adatom on the surface.

(4) Aggregation. If two particles come to occupy neighboring sites, they stick irreversibly and form an island. Islands are assumed to be immobile. 
We first analyse the results when desorption is negligible $\left(\tau_{e}=\infty\right)$. Fig 2 shows the evolution of the island density for a coverage of $15 \%$ (which we have checked is also the maximum island density, see also [3]) as a function of the rescaled frequency $f / d$. We see the three regimes predicted by our simple analysis. In particular, the relation $N_{i} \sim f^{-1 / 2}$ is well verified in the intermediate regime. The inset shows that the ratio of the low and high frequencies island densities scales as $d^{\chi}$, as predicted.

When desorption is important, the agreement is also very good. Fig 3 shows that the relation $N_{i}^{e} \sim f^{-1}$ is well verified in the intermediate regime. As in the case of complete condensation, the inset in Fig 3 shows that the ratio of island densities in the low and high frequencies regimes scales as $d$.

Summary, Perspectives : As anticipated, we have shown that it is not possible to interpret growth experiments under a chopped flux in the framework of usual growth models [3 5] which assume a continuous flux. For example, the relation of the saturation island density to the experimental parameters (flux, diffusion coefficient) depends on the regime, i.e. on the frequency of the chopped flux.

What kind of information can be derived from flux-modulated experiments for usual deposition techniques such as Molecular Beam Epitaxy? We note that modulation of the flux can be achieved rather easily by intercalating a rotating disk with a slit close to the sample. The order of magnitude of the frequencies required to observe the three regimes is reasonable. For example, in Stroscio's Fe/Fe(001) experiments [14], at room temperature, $N \sim 710^{12} \mathrm{~cm}^{-2}$ and $D \sim 710^{-12} \mathrm{~cm}^{2} \mathrm{~s}^{-1}$ then $f_{\text {high }} \sim 4 D N \sim 200 \mathrm{~Hz}$ and $f_{\text {low }}=d f_{h} \sim$ $2 \mathrm{~Hz}$ for $d=0.01$. In the case of complete condensation, measuring the saturation island density $N_{\text {sat }}$ as a function of frequency can help checking the growth conditions. First, $N_{\text {sat }}$ should show a sharp decrease for frequencies close to $1 / \tau_{m}$. Second, the ratio of the densities in the two extreme regimes should scale as $d^{\chi}$ if aggregation is indeed irreversible. We notice that this test - which is similar to the $N_{\text {sat }}$ versus flux test [3, [7,8, 15] - can be carried out experimentally without changing the flux, or even without knowing it. The 
effects of reversible aggregation should be addressed in future studies. The intermediate regime is also interesting since $N_{\text {sat }}$ is independent of the diffusion constant (and therefore of temperature). In growth experiments carried under a continuous flux, this constant density may only be observed for growth in presence of trapping impurities or reconstructed surfaces. In the case of growth in presence of important desorption, we speculate that this method allows to measure the evaporation time in-situ. By using reflection-high-energyelectron diffraction (RHEED) one could in principle measure a characteristic time needed for growing a monolayer, say a quarter oscillation. Although RHEED oscillations are difficult to interpret in detail, we anticipate that this characteristic time should depend strongly on the modulation frequency in the neighborhood of $f \simeq 1 / \tau_{e}$. Therefore, studying the evolution of this characteristic time as a function of the chopping frequency may lead to an estimate of $\tau_{e}$. 


\section{REFERENCES}

* Corresponding author. e-mail: jensen@dpm.univ-lyon1.fr

[1] M. A. Herman and H. Sitter, Molecular Beam Epitaxy, Springer-Verlag (Berlin, 1989).

[2] M. Lagally, Physics Today 46(11), 24 (1993) and references therein.

[3] J. Villain, A. Pimpinelli, L.-H. Tang, and D. E. Wolf, J. Phys. I France 2, 2107 (1992)

[4] J. A. Venables, G. D. T. Spiller, and M. Hanbücken, Rep. Prog. Phys. 47, 399 (1984).

[5] S. Stoyanov and D. Kaschiev, Current Topics in Mat. Science vol. 7, Ed. E. Kaldis, (North-Holland, 1981)

[6] P. Melinon, V. PAillard, V. DUpuis, A. PEREZ, P. JENSEn, A. HOAREAU, J.P. PEREZ, J. TUAILLON, M. BROYER, J.L. VIALLE, M. PELLARIN, B. BAGUENARD and J. LERME, J. of Phys. I (France) 3, 1585 (1993); A. PEREZ, P. MELINON, V. DUPUIS, P. JENSEN, B. PREVEL, J. TUAILLON, L. BARDOTTI, C. MARTET, M. TREILlEUX, M. BROYER, M. PELLARIN, J.L. VIALLE, and B. PALPANT, J. of Physics D 301 (1997)

[7] L. Bardotti, P. Jensen, M. Treilleux, A. Hoareau and B. Cabaud, Phys. Rev. Lett. 74, 4694 (1995); Surf. Sci. 367276 (1996)

[8] H. Brune, H. Röder, C. Borragno, and K. Kern, Phys. Rev. Lett. 73, 1955 (1994)

[9] M. C. Bartelt, S. Gunther, E. Kopatzi, R. J. Behm and J. W. Evans, Phys. Rev. B 53, $4099(1996)$

[10] P. Jensen, H. Larralde and A. Pimpinelli, Phys. Rev. B 55, 2556 (1997)

[11] G.S. Bales and D.C. Chrzan, Phys. Rev. B 50, 6057 (1994)

[12] P. Jensen, A.-L. Barabási, H. Larralde, S. Havlin, and H. E. Stanley, Nature 368, 22 (1994); Phys. Rev. B 50, 15316 (1994) 
[13] L.-H. Tang, J. Phys. I France 3, 935 (1993); J.W. Evans and M.C. Bartelt, J. Vac. Sci. Technol. A 12, 1800 (1994); J.G. Amar, F. Family, and P.-M. Lam, Phys. Rev. B 50, 8781 (1994). For a review, see A.-L. Barabasi and H. E. Stanley, Fractal Concepts in Surface Growth (Cambridge University Press, 1995) and J. Villain and A. Pimpinelli Physique de la Croissance Cristalline (Eyrolles, 1995) (English edition to be published by Cambridge University Press, 1997)

[14] J. A. Stroscio and D. T. Pierce, J. Vac. Sci. Technol. B 12, 1783 (1994)

[15] S. Gnther, E. Kopatzi, M. C. Bartelt, J. W. Evans and R. J. Behm, Phys. Rev. Lett. 73, 553 (1994); J.-K. Zuo, J. F. Wendelken, H. Dürr and C. L. Liu, Phys. Rev. Lett. $72,3064(1994)$ 


\section{FIGURES}

FIG. 1. Schematic illustration of the chopped flux (bold) and the corresponding evolution of the monomer density (dashed line). $F_{i}$ and $F_{a v}$ refer to the instantaneous and average flux respectively.

FIG. 2. Monte Carlo simulation of the island densities as a function of the rescaled chopping frequency $\mathrm{f} / \mathrm{d}$ in the case of complete condensation. The island densities correspond to a surface coverage of $15 \%$, an instantaneous flux $F_{i}=10^{-7}$ and a diffusion constant $D=1 / 4$. Each curve corresponds to a different value of $d: d=.5$ (circles), $d=.1$ (squares), $d=.01$ (diamonds), $d=.001$ (triangles). The solid line has a slope of $-1 / 2$. The inset shows the dependence of the high frequency island density on $d$. The solid line is a fit to the data and has a slope 0.36 , in excellent agreement with the predicted slope $(\chi=0.36)$.

FIG. 3. Computer simulation of the island densities as a function of the rescaled chopping frequency f/d when growth is limited by evaporation. The island densities correspond to a deposition time $F_{a v} t=0.77$, an instantaneous flux $F_{i}=10^{-7}$, a diffusion constant $D=1 / 4$ and an evaporation time $\tau_{e}=100$. Each curve corresponds to a different value of $d d=.5$ (left triangles), $d=.1$ (squares), $d=.01$ (circles), $d=.003$ (triangles up), $d=.001$ (diamonds). The solid line has a slope -1 . The inset shows the dependence of the high frequency island density on $d$. The solid line is a fit to the data and has a slope 0.97 , in excellent agreement with the predicted slope of 1. 


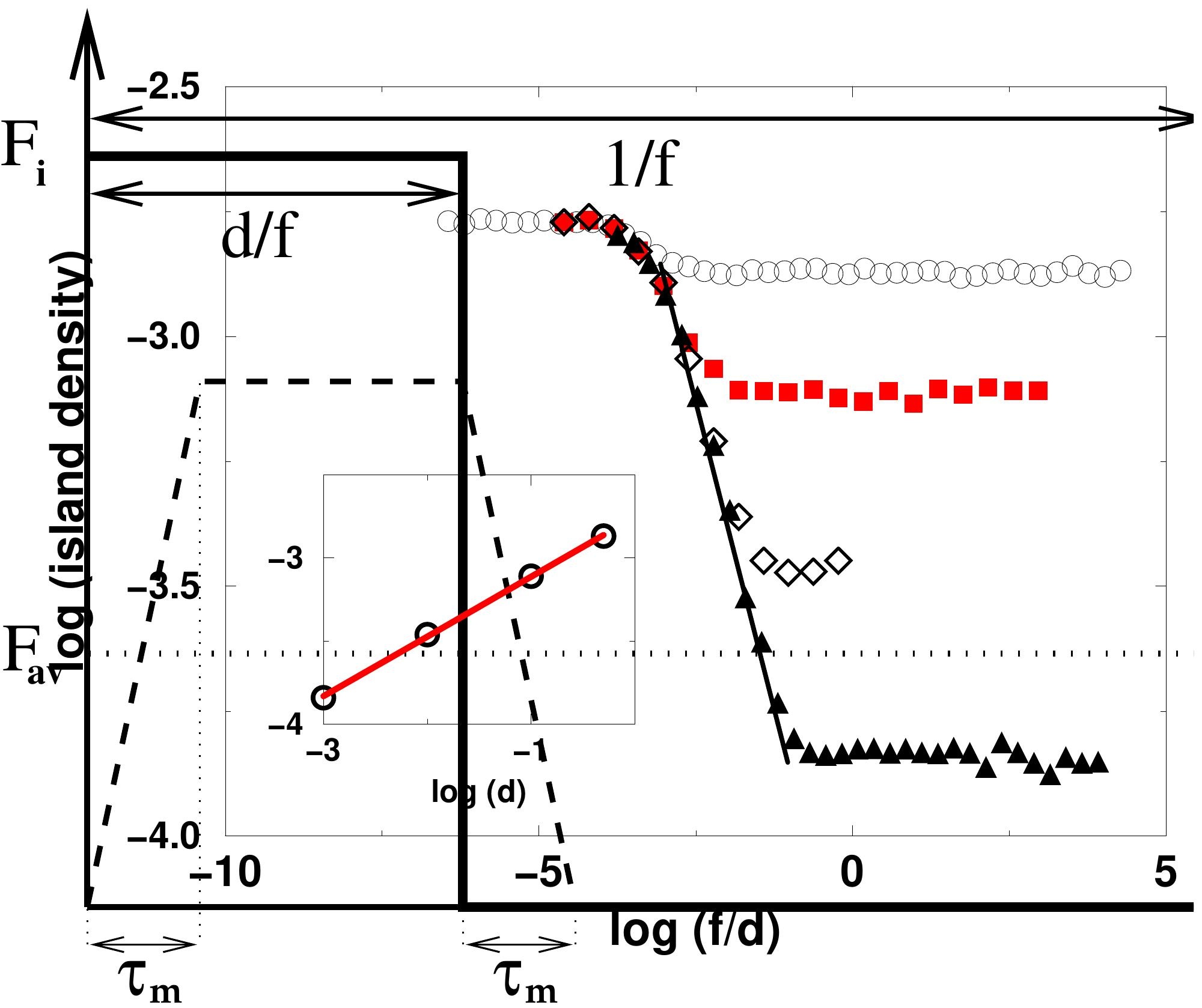




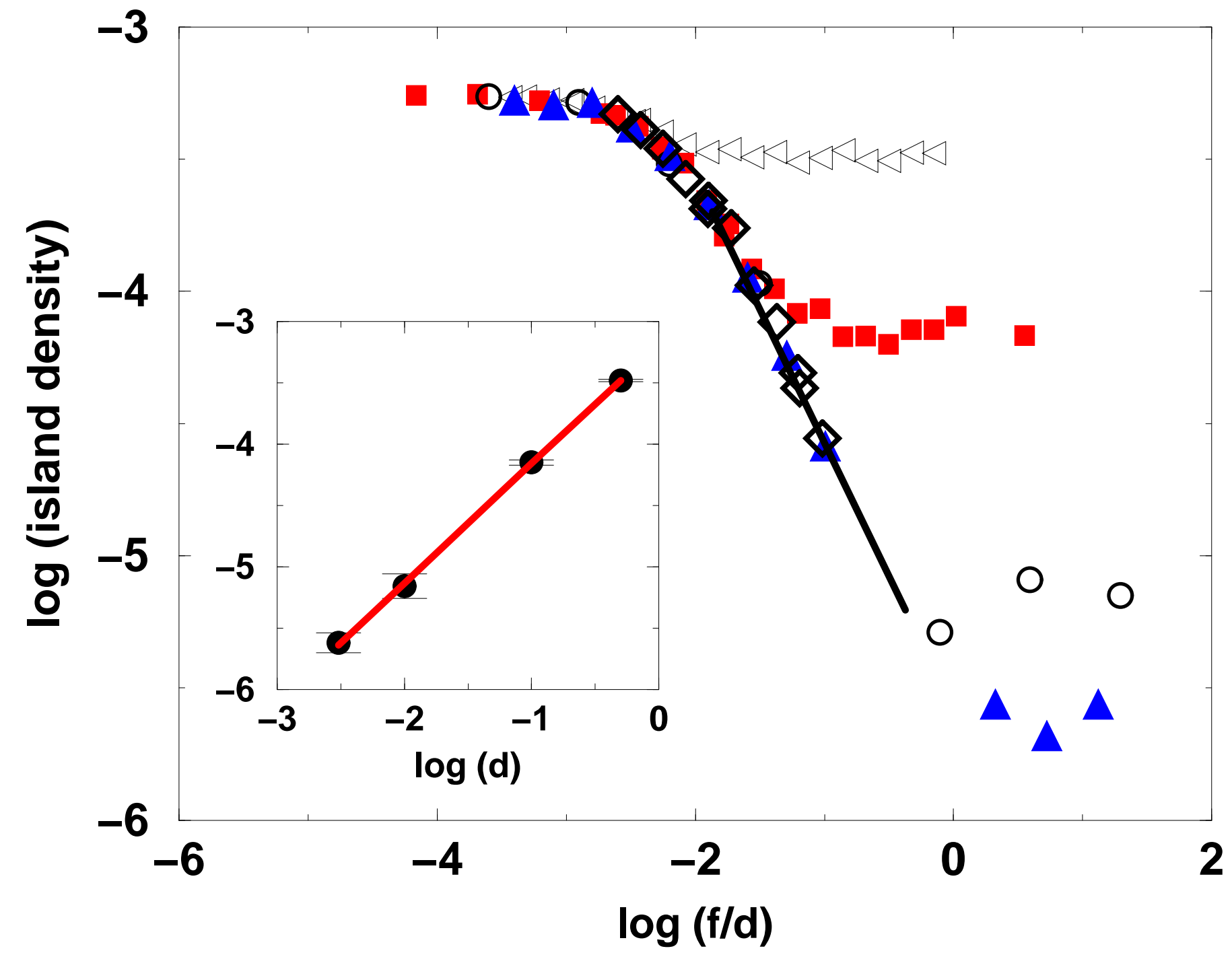

